\title{
Roles y prácticas educativas en el proceso de inclusión de estudiantes con dificultades de comunicación
}

\section{Educational roles and practices in the process of inclusion of students with communication difficulties}

DOI: $10.46932 /$ sfjdv2n4-092

Received in: March 1st, 2021

Accepted in: May 30th, 2021

\author{
María Julieta Maldonado Figueroa \\ Dra. En Educación \\ Escuela Normal Estatal de Especialización \\ Callle Colosio, s/n. Providencia, Sonora, México \\ mjmalfig@gmail.com \\ María Angélica Quiroz Leyva \\ Mtra. En Educación Especial \\ Escuela Normal Estatal de Especialización \\ Callle Colosio, s/n. Providencia, Sonora, México \\ E-mail: quirozleyva@gmail.com \\ Gamboa Robles Marco Antonio \\ Dr. En Planeación y Liderazgo Educativo \\ Escuela Normal Estatal de Especialización \\ Callle Colosio, s/n. Providencia, Sonora, México \\ E-mail: enee.mgamboa@gmail.com \\ Dinorah Burgos Corral \\ Lic. En Educación Especial \\ Escuela Normal Estatal de Especialización \\ Callle Colosio, s/n. Providencia, Sonora, México \\ E-mail: eneesonca1@gmail.com
}

\section{RESUMEN}

Las prácticas docentes inclusivas para abordar los problemas de lenguaje más comunes en una escuela regular y su proceso de intervención lo realiza la Unidad de Servicio de Apoyo a la Educación Regular (USAER), desde el área de comunicación, por lo cual, el estudio se perfila a las estrategias didácticas que favorecen el nivel fonológico en alumnos que presentan dislalia funcional. Las prácticas docentes del especialista logopeda, se traducen en acciones inclusivas que atienden el trastorno lingüístico como una necesidad actual de relevancia, sobre todo en aquellos alumnos que lo presentan por rotacismo, es decir, con dificultad con el fonema $r$ en todas sus dimensiones, siendo éste, el de mayor grado de dificultad, debido al proceso de maduración fisiológica. Por lo tanto, favorecer y proporcionar herramientas que permitan minimizar y posiblemente erradicar dichas dificultades, implica indagar acerca de la estrategia inclusiva que permitirá de una manera atractiva e innovadora, estimular la producción del lenguaje. Para esto, el diseño de programas específicos, aplicación de diversas pruebas estandarizadas para evaluar el lenguaje, entrevistas, cuadernos de seguimiento para casa y registros anecdóticos, fungen como instrumentos de recogida de datos eficaz para elegir la estrategia de intervención efectiva para el estudio en cuestión. 
Palabras clave: Práctica docente, Práxias lingüisticas, inclusión educativa.

\begin{abstract}
The inclusive teaching practices to address the most common language problems in a regular school and its intervention process is carried out by the Unit of Support Service to Regular Education (USAER), from the area of communication, therefore, the study is profiled to didactic strategies that favor the phonological level in students who present functional dyslalia. The teaching practices of the speech therapist specialist, are translated into inclusive actions that address the linguistic disorder as a current need of relevance, especially in those students who present it by rotacism, that is, with difficulty with the phoneme $r$ in all its dimensions, this being the one with the highest degree of difficulty, due to the physiological maturation process. Therefore, favoring and providing tools that allow minimizing and possibly eradicating such difficulties, implies investigating about the inclusive strategy that will allow, in an attractive and innovative way, stimulating the production of language. For this purpose, the design of specific programs, the application of various standardized tests to evaluate language, interviews, home follow-up notebooks and anecdotal records, serve as effective data collection instruments to choose the effective intervention strategy for the study in question.
\end{abstract}

Key words: Teaching practice, linguistic praxis, educational inclusion.

\title{
1 INTRODUCCIÓN
}

Las prácticas docentes inclusivas refieren la sistematización de acciones que generen ambientes de aprendizaje, facilite y provea de recursos materiales e intelectuales, y elimine las Barreras para el Aprendizaje y la Participación (BAP), considerando intereses, gustos, y ritmos de aprendizajes en la diversidad del alumnado que se atiende. Para efectos del presente estudio, el seguimiento se dió paso a paso, donde se atendió a los estudiantes a través de sesiones de trabajo grupal, sub grupal e individual, utilizando una estrategia diversificada con juegos tradicionales adaptados al área del lenguaje, permitiéndole al alumno interactuar con material didáctico concreto y llamativo, así como también las tareas que se establecieron para realizar en casa.

Durante la implementación de dicha estrategia, se presentaron algunas dificultades que fueron causantes de modificaciones y adecuaciones imprevistas a las actividades planeadas, entre las cuales se encuentra la falta de espacios acordes para cada actividad, carencia de tiempo por acciones cocurriculares que se encontraban fuera de nuestro alcance, poca colaboración por parte de los padres de familia, así como inasistencias de los alumnos por diversas razones injustificadas, sin embargo, se buscaron soluciones que volvieran este proceso un poco más favorecedor como la optimización de tiempos mediante la modificación de horarios de atención temporal.

El contenido del estudio potencializa la práctica inclusiva en condiciones reales de trabajo de dos escuelas primarias que cuentan con el servicio de la USAER. Presenta una problemática específica del 
área de comunicación, dando respuesta de las posibles soluciones que cubren de manera adecuada las necesidades presentes.

\section{PROBLEMATIZACIÓN}

El lenguaje se integra por su forma, contenido y uso, lo cual se desarrolla por medio de sus cuatro dimensiones que son el nivel fonológico, sintáctico, semántico y pragmático. Cuando alguno de estos aspectos se encuentra afectado, se da paso a un problema en esta área, además en la comunicación, lo que se considera como la dificultad para expresarse o interactuar, presentándose desde la obstaculización para articular los fonemas, manifestándose mediante la omisión, sustitución o modificación de éstos, hasta el impedimento de comprender o utilizar el lenguaje correctamente de acuerdo a la edad que presente el individuo. Haciendo énfasis en el nivel fonológico, se define como el aspecto por el cual se articulan los sonidos de una lengua, mediante el empleo de su punto y modo, con el fin de formar palabras u oraciones. Dentro de sus dificultades se encuentran distintos tipos de alteraciones que varían en cuanto a su procedencia y los grados de severidad. Entre los principales y los que se observan con mayor frecuencia en las instituciones educativas, se encuentran la dislalia, disfasia, disglosia, disartria, disfemia y disfonía. Estos podrían causar dificultades en la interacción y en ciertas situaciones provocar un asilamiento del alumno en relación con sus compañeros, bajo rendimiento escolar que en algunos casos desencadena en un problema de aprendizaje, entre otras situaciones.

$\mathrm{Al}$ considerar que la muestra del estudio son alumnos de cuarto grado de primaria con nueve años de edad, que algunos presentan un desfase cronológico, además sus dificultades lingüísticas ya son percibidas por ellos mismos y por sus compañeros, impacta de manera negativa en su desenvolvimiento en los diversos contextos con sus iguales. Ante tal situación, con el fin de atender a las necesidades escolares planteadas y dar seguimiento para aportar en cuanto a su desarrollo linguístico, como parte del quehacer del docente inclusivo, desde el área de comunicación se implementan estrategias didácticas diversificadas, que fortalece el desarrollo del nivel fonológico. Al ser dinámicas lúdicas de interés y fácil de llevarlas a cabo, promete logros significativos en la articulación, mayor seguridad y motivación personal, así como mayor fuerza en acciones indirectas que benefician al lenguaje, como lo son las praxias de respiración y soplo. El trabajo colaborativo entre los actores involucrados en la educación de los sujetos, juegan un papel indispensable en la estrategia diversificada de la práctica inclusiva de los especialistas de educación especial, exclusivamente desde el área de comunicación. 


\section{MARCO TEÓRICO}

Dentro del nivel fonológico se presentan distintos tipos de dificultades en cuanto a la articulación, estos ocasionados por diversas causas, las cuales se describen para posteriormente ubicar y centrar el tema en cuestión en una de estas clasificaciones, tomándolo como punto de partida para comparar las características con la realidad (Leong, 2004). La intervención se basa en la implementación de estrategias didácticas como prácticas inclusivas del área de comunicación con los alumnos sujetos de estudio. Correspondientemente se hacen los ajustes razonables pertinentes, con el fin de cubrir las necesidades educativas específicas de la muestra.

El lenguaje, siendo la herramienta que utiliza el ser humano para comunicarse e interactuar con el medio que le rodea, en el contexto escolar y áulico, se observa como un instrumento esencial, puesto que a través de éste se proporciona principalmente los contenidos, además, se utiliza para llevar a cabo la mediación, ya sea en actividades académicas o en situaciones imprevistas que se presenten tanto dentro como fuera del aula. Según Calderón (2005), éste es dividido en cuatro componentes que se agrupan de acuerdo a la forma, el contenido y el uso que se les da, esto con el fin de hacer que su estudio sea más preciso, puesto que, la información de cada uno en cuanto a su desarrollo permite que en caso de existir alguna dificultad, sea más factible su detección, clasificación e intervención.

Estos cuatro componentes son el fonológico, semántico, sintáctico y pragmático. De manera general, se puede decir que el fonológico hace alusión a la forma del lenguaje, ya que este aspecto muestra la manera correcta en la que cada vocal o consonante debe ser articulada conforme a su sonido. Por otra parte, el semántico se integra por el contenido, explicando los aspectos del significado o interpretación de una palabra. En cuanto al sintáctico, se puede decir que también pertenece a la forma, puesto que es el componente que nos señala la estructuración. Por último, el pragmático se considera como el uso del lenguaje, en el cual al interactuar se ponen en práctica los tres componentes mencionados anteriormente.

En las instituciones educativas es común encontrar situaciones en las cuales los alumnos presentan dificultad en algunos de estos cuatro componentes, siendo detectados por los diversos actores involucrados, quienes se relacionan diariamente con ellos. Es importante delimitar en qué aspecto es donde radica el problema, para poder actuar lo más pronto posible. De acuerdo a la muestra, los mayores inconvenientes se sitúan en el nivel fonológico, detectándose omisión, sustitución o distorsión de los fonemas al hablar, siendo notorio al entablarse una conversación o al participar en clase.

Es por lo anterior que se considera indispensable conocer el desarrollo fonológico, lo cual se entiende como la integración progresiva de los fonemas, puesto que nos permite identificar y comparar cuando un estudiante muestra un desfase cronológico. Jiménez y Obispo (2006), opinan que cada uno de estos fonemas son adquiridos a los largo de los años de la vida del niño, pasando por diferentes etapas. 
Este es un proceso que inicia desde los primeros meses de edad y conforme la edad cronológica se encuentran distribuidas las vocales y consonantes, las cuales se van adquiriendo debido a que, a dicha edad el nivel madurativo de los órganos que intervienen se encuentran listos para articularlos. Cuando estos no logran ser dominados por el niño antes de los siete años de edad, se pudiera hablar acerca de una dificultad en la articulación. En condiciones reales de trabajo es muy común encontrarse, en ocasiones que no solamente influye el nivel madurativo de los órganos involucrados, sino que también intervienen diversos factores como el contexto y la estimulación que el alumno recibe, por lo cual es importante tomar en cuenta que dicha secuencia de desarrollo se basa en los resultados de una generalidad que sirve como punto de partida al docente que interviene académicamente en busca de solución.

Dentro de la institución es fundamental que se identifiquen las necesidades que el estudiante presenta, pues dicho lugar representa para algunos un espacio en el que se favorece la ampliación de la socialización, siendo el único contexto al que se enfrentan después del núcleo familiar, además en éste se pueden detectar situaciones no antes previstas por los padres, es por ello que los docentes cumplen un papel indispensable.

Conforme se van desarrollando las sesiones de clases dentro de las aulas, los docentes identifican y canalizan a aquellos alumnos que según su criterio cuentan con aspectos que les provocaban una disfunción al querer compartir información, ya sea a ellos mismos o a sus compañeros, así como timidez al participar en clases, poca interacción y en algunas ocasiones logran identificarlo debido a que este repercute en la escritura. Los problemas de lenguaje más comunes encontrados en la muestra del estudio, es en el peor de los casos la omisión de fonemas al hablar, repercutiendo en su desempeño escolar dentro del grupo, afectando también la participación en actividades grupales, debido a las dificultades de articulación.

Como acciones docentes de los procesos inclusivos que llevan los profesionales de la educación involucrados en este estudio, es el maestro de grupo el encargado de canalizar la situación al área especializada correspondiente. Aquí es donde entra la función especializada del experto en audición y lenguaje. El maestro de comunicación remite de manera específica al diagnóstico con el que cuentan dichos estudiantes atendidos en ésta área de educación especial, que para efectos reales del estudio, se presentan cuadros de dislalia, lo que se considera como un trastorno del lenguaje que consiste en la dificultad articulatoria de uno o varios fonemas, los cuales sustituye, omite o distorsiona, sin encontrarse comprometido con una afectación cerebral o con los órganos que intervienen en su producción.

En este sentido, la práctica inclusiva del maestro de comunicación en condiciones reales de trabajo, realiza como primer momento la identificación del diagnóstico, el cual determina la dificultad más frecuentes presentada por los estudiantes, ésta es detectada a través de la observación del lenguaje oral y 
la aplicación de diversos instrumentos de evaluación, en los cuales pone en práctica la utilización de todos los fonemas para determinar en cuáles se ubicaba el problema. Para realizar un buen diagnóstico es necesario tomar en cuenta la situación del alumno, así como el desarrollo fonológico, para verificar si se encuentra en proceso de adquisición o si definitivamente, se trata de un caso de dislalia y no de otro tipo de trastorno que se relacione con alguna afectación en otras áreas.

Los estudiantes sujetos de estudio, presentan un desfase, lo que ocasiona que omitan, distorsionen o sustituyen el fonema en cuestión y al presentar entre nueve y diez años de edad, se ubican fuera del rango de adquisición, convirtiéndolo por consiguiente en un problema de lenguaje. Con base a la indagación mediante entrevistas a los padres de familia, diagnósticos emitidos por un médico y las evaluaciones de lenguaje, ninguno de los casos presenta malformaciones físicas, afectaciones cerebrales, así como se descartó la posibilidad de una dislalia evolutiva considerándose su edad cronológica, ubicándose por la USAER en una dislalia funcional.

Cabe destacar que una acción inclusiva del maestro de comunicación, necesaria para recabar información se tornó a la implementación de una guía de observación enfocada en la interacción de los estudiantes, tanto con sus iguales como con sus maestros, con el fin de conocer si dicho problema de lenguaje interfiere en cuanto a sus relaciones interpersonales dentro de la institución. Se realizaron intervenciones grupales y se destinaron espacios de observación tanto en el aula como en los momentos de recreación. La estrategia de intervención tiene como fin desarrollar y favorecer en el estudiante los aspectos en los que presentó dificultad, con la intención de mejorarlos, dotándolo de herramientas para beneficiar su aprendizaje. Es por ello que una estrategia permanente fue poner en práctica la respiración, el soplo, realizar movimientos linguales, así como involucrar las piezas dentales, mejillas, paladar, además de practicar el sonido aislado del fonema e integrarlo en palabras, oraciones y emitirlo mediante actividades de manera espontánea; llevándose a cabo con estrategias diversificada de enfoque inclusivo.

Bajo el enfoque inclusivo que el docente del área de comunicación realiza su práctica docente, la estrategia diversificada consistió en la implementación de acciones fuera de lo común, donde se trabajó de manera distinta a lo que los estudiantes estaban acostumbrados, permitiéndoles y facilitándoles la interacción con material didáctico creativo, diversas formas de aprender, así como distintas maneras de abordad el contenido, donde el estudiante se sentía motivado por participar (SEP, 2012). Un factor importante fue el conocer los gustos e interés del alumno, así como sus características en cuanto a ritmos y estilos de aprendizaje, para seleccionar la estrategia más favorable. Se consideró el juego como una alternativa viable y diversificada de atención, debido a que a través de este se cubren necesidades de interés, dotándoles de un espacio de relajación en el cual al crear un ambiente de confianza motiva y genera disposición ante la realización de los ejercicios pertinentes para favorecer sus dificultades. Por lo 
tanto, la pretensión de la estrategia fue incitar al alumno a interactuar, compartir opiniones, ampliar su repertorio lingüístico, entre otras situaciones que le permitieran poner en práctica su lenguaje oral, y que a su vez mediante adaptaciones a juegos convencionales practicara la articulación, además de ejercicios de soplo, respiración, linguales y labiales (Fernández, 2014).

Otro aspecto esencial de la inclusión educativa es trabajar en redes de colaboración entre todos los involucrados en el proceso educativo del alumno, en este sentido el trabajo colaborativo entre los actores fungió como parte de la estrategia de intervención del área de comunicación. Según Puigdellivol (2001) mantener una relación estrecha con la familia del estudiante en la cual se establezca un lazo colaborativo, favorecerá a la ampliación del aprendizaje escolar, por lo cual la escuela y el hogar no deben manejarse como dos contextos aislados. Es sabido que el apoyo que se brinda por los padres favorece enormemente los logros escolares de los niños, pues se refuerza lo aprendido en las clases, además de darse seguimiento a las actividades.

Dentro de los servicios de apoyo y sobre todo del área de comunicación, se considera este uno de los puntos más importantes, debido al trabajo itinerante que realiza el docente de auditiva y de lenguaje, asistiendo solamente un día a la semana a cada institución. Es por ello que es relevante entablar vínculos colaborativos con los padres de familia, los cuales deben ser asesorados para continuar durante toda la semana aplicando las actividades proporcionadas por el docente SEP (2011). Por otra parte el aspecto del lenguaje requiere de trabajo constante e ininterrumpido pues los resultados se dan paulatinamente, entonces, al no llevarse un seguimiento en las acciones a realizar, difícilmente se muestren resultados favorables.

\section{METODOLOGÍA}

Se presenta un estudio cualitativo de corte descriptivo, aplicado a una muestra de 60 alumnos de entre nueve y diez años de edad, que cursan cuarto grado de primaria, divididos en dos escuelas urbanas diferentes que en coincidencia cuentan con atención de la Unidad de Servicio de Apoyo a la Educación Regular (USAER). Las prácticas docentes inclusivas del presente estudio, reflejan el quehacer exclusivo del especialista en el área de comunicación, siendo éste un elemento experto del área auditiva y de lenguaje que forma parte del equipo interdisciplinario de educación especial.

\section{PRESENTACIÓN Y DISCUSIÓN DE RESULTADOS}

Para poner en marcha la práctica inclusiva del maestro de comunicación, como primera instancia tendrá que llevar un proceso de detección y evaluación de los problemas de lenguaje, y todo lo que ello implica. Uno de los primeros pasos para la intervención claramente es la detección, lo cual hace referencia 
al acto de identificar o descubrir algo mediante la indagación de datos, utilizando diversas técnicas e instrumentos. Para el caso específico del presente estudio, se hablará de percibir a aquellos estudiantes que dentro y fuera del aula muestran una dificultad al articular los fonemas al hablar, lo cual podría repercutir en diversas áreas. Para ello fue necesario llevar a cabo una serie de pasos antes de comenzarse con el proceso de evaluación que determinara un problema de lenguaje.

$\mathrm{Al}$ inicio del ciclo escolar se llevaron a cabo observaciones grupales, ingresando a las aulas con el fin de interactuar con los estudiantes, conocer sus características y percibir su lenguaje oral. Para ello se realizaron algunas dinámicas, las cuales incitaban a que los alumnos participaran, facilitando con esto la detección de alguna dificultad. Entre dichas actividades se encontraban la lectura de cuentos, el canto de algunas melodías infantiles, juegos con trabalenguas, preguntas espontáneas sobre sus gustos y preferencias, también contaban historias acerca de sucesos que les ha tocado vivir o presenciar. Todo esto dio paso a un mayor acercamiento con los niños, donde se fueron observando aquellos casos mayormente notorios, creándose un listado de manera informal para posteriormente avanzar al siguiente nivel del proceso e ir descartando.

Cabe destacar que dentro de este primer paso intervinieron los maestros de grupo regular, quienes al encontrarse en constante convivencia con los estudiantes, facilitaron el proceso de detección, canalizando al servicio de apoyo a todos aquellos estudiantes que durante las clases percibían con algún problema de articulación. La comunicación entre los docentes fue de gran relevancia, puesto que compartían toda la información que durante la semana lograron rescatar.

El siguiente paso consistió en llevar a cabo la observación individual; una vez que se realizó el listado de alumnos con posible problema de lenguaje, en esta dinámica se les pedía que asistieran al aula de la USAER, donde se le hacían preguntas acerca de su vida cotidiana ya sea sobre sus gustos, sus intereses, la escuela, su familia, actividades de recreación dentro y fuera de la institución, entre otras situaciones que en el momento se iban suscitando. Esto se daba de manera espontánea, lo cual genera un ambiente de confianza donde el alumno al final lograba expresarse libremente a diferencia que al inicio, cuando ingresaba al salón percibiéndose tímido. Esto permitió identificar con mayor detalle lo observado en el aula, concluyendo con esto el proceso de detección, para continuar con la evaluación.

Una vez que se formuló la lista de alumnos detectados, se comenzó el proceso de evaluación inicial, realizándose de manera individual en el aula de la USAER, mediante la aplicación de un instrumento propio del área de comunicación basado en una recopilación de siete aspectos a evaluar, dentro de los cuales se encuentran: interacción comunicativa, discriminación fonológica, memoria auditiva, preguntas de razonamiento lógico, corpus, descripción a través de una lámina con imagen y la comprensión lectora. 
Con base a estos indicadores se realizaron a los alumnos preguntas básicas acerca de información personal y gustos, repetición de palabras para verificar si lograba distinguir los fonemas, repetición de oraciones largas y cortas para percibir su alcance de retención, cuestionamientos sencillos en los que se esperaba que emitieran respuestas de manera coherente y lógica, la emisión del nombre de objetos presentados en imágenes a través de tarjetas para detectar dificultades a nivel fonético, conocer su nivel de observación y detección de objetos mediante la implementación de descripción o narración de láminas, además la presentación de cuentos corto con referentes visuales, emitiendo lo que logró entender para posteriormente responder a preguntas de análisis de la historia.

Después se realizó la detección de las Barreras para el Aprendizaje y la Participación (BAP), conforme a los resultados que arrojó dicha evaluación, así como analizándose los diversos aspectos rescatados de diagnósticos médicos o entrevistas con los padres de familia. Una vez que se contaba con esta información se llevó a cabo la realización de los informes iniciales y se determinó si el estudiante sería anexado al registro de atención. En el caso de ambos estudiantes los resultados fueron muy similares, atendiendo a la mayor parte de los indicadores sin ninguna dificultad, solamente en el apartado enfocado en la articulación de fonemas, lo cual fue plasmado en sus respectivos informes.

Toda la documentación incluyendo el diseño de un plan anual de tratamiento, se anexó a su expediente, con la sugerencia de trabajarlos durante todo el ciclo escolar. Este consistió en cuatro listados de actividades y/o sugerencias de acción, referentes a cada una de los cuatro componentes del lenguaje, donde se marcaban las que correspondieran a las necesidades de cada alumno y que posteriormente se desarrollaron durante las sesiones.

También se aplicó el test de prueba de articulación de fonemas (PAF) el cual está específicamente diseñado para estudiantes que presentan dislalia, el cual contenía algunos aspectos similares a la evaluación inicial, esto con el fin de recabar más datos que permitieran intervenir de una manera mayormente adecuada, pretendiendo atender solamente las necesidades del estudiante. En comparación con la anterior evaluación, éste solamente se integraba por apartados relevantes y enfocados a los problemas de articulación, puesto que el otro instrumento se encargó de evaluar los cuatro componentes del lenguaje. El proceso diseñado es uno de los pasos clave que permitirá realizar una correcta intervención que dará paso a una enseñanza asertiva, elemento clave de las prácticas inclusivas efectivas.

De acuerdo a los resultados obtenidos, se rescata con mayor énfasis una necesidad de reforzamiento constante en cuanto al a espiración nasal y bucal, para ejercitar mayor intensidad en el soplo, a discriminación entre una exhalación fuerte y una débil, también del dominio de este en torno a la direccionalidad. Por otra parte, el control de movimiento de lengua y labios, para promover agilidad, flexibilidad, control voluntario y logre articular todos los fonemas con el posicionamiento de la lengua, 
sobre todo para producir el fonema /r/, fuerza, duración y dominio del soplo. Además discriminar auditivamente palabras y letras.

El diseño del programa específico de lenguaje enfocado a los alumnos que presentan rotacismo, siendo éstos de primaria alta se negaban por cohibición a realizar el ejercicio, por lo que se buscó la manera de trabajar mediante una estrategia que no les provocara dicho sentimiento. Para cubrir las necesidades específicas de éstos, se planearon ejercicios basados en el fonema en cuestión con el fin de favorecer a su correcta articulación, lo cual se lograría indagando en aquellas actividades en las cuales se tomara en cuenta los aspectos que los incitaran a participar de manera voluntaria. Otro aspecto que se consideró, fue el gusto que mostraron por aquellas dinámicas en las que se involucraban otros aspectos tales como la concentración, la memoria, el movimiento, la destreza, la rapidez y todas aquellas cuestiones que los ponían en acción, compitiendo de manera sana. Esto les producía la necesidad de participar y realizar las encomiendas sin sentirse avergonzados, haciéndolo de manera espontánea.

La participación de los padres fue parte de la estrategia inclusiva en la práctica cotidiana del docente del área de comunicación. Al ser una generalidad coincidente la reducida asistencia de los padres, se buscaron distintas alternativas, optándose por la creación de cuadernos de seguimiento, en los cuales se les brindaron a los estudiantes diversas actividades con referencia al fonema en cuestión. Dicha dinámica al ser idealizada se percibió como favorable debido a que se visualizó como una práctica mediante la cual el alumno reforzaría los distintos tipos de práxias en su hogar, así como ejercitaría la correcta articulación involucrando mediante el apoyo de la observación como ejemplo al padre o la madre de familia. Dicho cuaderno de seguimiento constaba de actividades temporales específicas que hacían referencia al fonema que se trabajó durante la sesión, las cuales involucraban la articulación directa mediante la repetición, siendo cambiantes cada semana. Por otra parte, se encontraban ejercicios permanentes, que consistían en práxias linguales, labiales, de respiración, absorción y soplo, presentadas a través de imágenes con descripciones cortar y concretas, las cuales se pretendía que se realizaran todos los días a lo largo de todo el ciclo, ya que estas no eran cambiantes.

De acuerdo a lo descrito anteriormente, se puede decir que a pesar de que la participación de los padres dentro del entorno educativo y el desarrollo del aprendizaje de los estudiantes, es indispensable, se continúa careciendo de la colaboración. Dicha falta de relación entre el docente y los progenitores de los alumnos representa una disruptiva entre lo que se quiere realizar y lo que se logra llevar a cabo, afectando en cuanto a los avances significativos que pudieran presentarse. Es indispensable crear conciencia acerca de la gran cantidad de beneficios que esta colaboración contrae, además de entablar relación con los distintos actores, que en su debido momento pudieran apoyar. 
La evaluación final cumple un papel muy importante en el proceso de atención dentro de los servicios de apoyo de educación especial, puesto que con este se rescata información con el fin de identificar si se presentaron avances o retrocesos en el alumno. Con estos se determina y se analiza si las actividades realizadas durante cierto periodo de tiempo dieron resultados favorables o no, qué es lo que resultó funcional que podría seguirse implementando o que cuestiones deben de ser modificadas, así como otras situaciones que deben tomarse en cuenta, tales como los espacios, los tiempos o los recursos didácticos implementados, lo cual tendrá como fin mejorar la labor docente. 56

Para presentar un esquema de los resultados, cabe señalar que se llevó a cabo la aplicación tanto del test PAF como la evaluación final de lenguaje, con los mismos apartados que conformaban el instrumento inicial. Dichos indicadores no fueron modificados, ya que se pretendía observar exactamente los mismos puntos, con el fin de que permitieran proporcionar de manera clara y rigurosa los avances o retrocesos que presentaron los estudiantes. En comparación con los primeros resultados, dicha evaluación arrojó aún dificultad en la articulación del fonema /r/ fuerte y suave, manejándolo por sustitución, sin embargo hubo avances significativos en el aspecto actitudinal, mostrándose mayor disposición al momento de realizar las repeticiones e intentar pronunciar palabras con dicho fonema. Se manifiesta cierto grado de adquisición del conocimiento en cuanto al posicionamiento correcto del punto y modo de articulación, con respectiva autocorrección.

Los resultados se registran en los informes finales, donde siguiendo el protocolo, se contempla primeramente los datos para identificar al alumno, la razón por la cual se encuentra canalizado al área de comunicación, posteriormente una breve descripción de las actividades que se trabajaron durante el ciclo escolar, después los avances significativos o retrocesos acompañados de situaciones relevantes observadas a lo largo del ciclo escolar tales como actitudes, inasistencias o actividades extraescolares en las que el alumno ha participado y que pudiera haber influido en los resultados, por último un listado con sugerencias para dar seguimiento. Cabe destacar que dicho informe es entregado a los padres de familia, por lo cual dentro de dichas sugerencias se plasman actividades con redacción sencilla y concreta para que éstos las trabajen durante las vacaciones.

\section{CONCLUSIONES}

Los escenarios inclusivos en el quehacer didáctico de los profesionales de educación especial, exigen conocimiento, manejo y dominio mínimos de las cuestiones específicas de su área. Para efectos del presente estudio, el maestro de comunicación, obligadamente deberá tener como base, la información de que los problemas de lenguaje son una dificultad que al igual que común es muy compleja, afectando a los estudiantes en cuanto a la fluidez del habla, repercutiendo en su estado anímico, actitudinal y 
pedagógico. Es importante entonces, que su práctica docente atienda esas necesidades, generando ambientes de confianza en los que se le permita al alumnado desarrollarse sin temor a ser causa de burlas, sobre todo cuando ya se encuentra en una edad en la que él mismo reconoce cuando comete errores.

Es por lo anterior que para un alumno con problemas de lenguaje a nivel fonológico se requieren de adaptaciones al currículo especializadas, donde se intensifique la práctica del fonema en el que presenta dificultad, dirigiendo todas las actividades hacia este, tomando siempre en cuenta sus gustos e intereses, donde se le permita desarrollarse sin temor a ser juzgado e incluso que lo haga por iniciativa propia, motivado por dinámicas que sean agradables y de interés para él. En este caso se implementó el juego como estrategia diversificada didáctica, realizándose adecuaciones de tipo metodológico, donde se practicó la actividad bajo las reglas de los juegos originales pero orientando el contenido hacia la producción de manera directa e indirecta.

Es además relevante establecer vínculos entre los distintos actores involucrados, ya que con esto se asegura un mayor logro y avances significativos. Los demás docentes y padres de familia, servirán como puentes para el aprendizaje del estudiante, siendo ellos quienes apoyen en situaciones de riesgo. Continuar con las actividades ininterrumpidamente será la clave para generar el aprendizaje deseado, pues la constancia del trabajo permitirá resultados óptimos a corto o a largo plazo en el estudiante. Es por ello que se sugiere continuar con la aplicación del programa específico escolar y de casa, debido a que la aplicación y seguimiento de ello comprueba buenos resultados y a largo plazo se podrían obtener avances mayormente significativos. 


\section{REFERENCIAS BIBLIOGRÁFICAS}

Calderón, M. (2005). Niños exitosos ¡Con pequeños pasos... grandes avances! Primera edición. Psicom editores. Colombia.

Jiménez, J. y Obispo, J. (2006). Superar las dificultades de lenguaje. Primera edición. La tierra hoy. España.

Leong, D. (2004). Tácticas: el uso del lenguaje. Primera edición. SEP. México.

Puigdellivol, I. (2001). La familia como agente de integración. Primera edición. Graó. Barcelona.

SEP. (2011). Plan de estudios. 1era. Ed. México.

SEP. (2012). Estrategias Específicas y Diversificadas para la atención educativa de alumnos y alumnas con discapacidad. 1era. Ed. México. 